\title{
Trastuzumab-Induced
}

\section{Myocardiotoxicity Mimicking Acute Coronary Syndrome}

\author{
K.B. Ribeiro ${ }^{a} \quad$ C.H. Miranda ${ }^{b} \quad$ J.M. Andrade ${ }^{c} \quad$ L.G. Galli \\ D.G. Tiezzic H.F. Oliveira ${ }^{a}$ F.E. Zola ${ }^{a} \quad$ G. Volpe \\ A. Pazin-Filho ${ }^{b}$ F.M. Peria ${ }^{a}$ \\ ${ }^{a}$ Clinical Oncology Division and ${ }^{b}$ Clinical Emergency and Cardiology Division, \\ Department of Internal Medicine, and 'Mastology and Gynecologic Oncology \\ Center, Department of Gynecology and Obstetrics, School of Medicine of \\ Ribeirão Preto, University of São Paulo - FMRP-USP, São Paulo, Brazil
}

\section{Key Words}

Breast cancer · Trastuzumab - Myocardiotoxicity · Acute coronary syndrome

\begin{abstract}
Trastuzumab is an important biological agent in the treatment of HER2-positive breast cancer, with effects on response rates, progression-free survival, overall survival and quality of life. Although this drug is well tolerated in terms of adverse effects, trastuzumabassociated myocardiotoxicity has been described to have an incidence of $0.6-4.5 \%$ and in rare cases, the drug can trigger severe congestive heart failure with progression to death or even mimic acute coronary syndrome with complete left bundle branch blockade. In this paper is reported a case of trastuzumab-associated myocardiotoxicity manifesting as acute coronary syndrome in a 69-year-old female. The patient is currently undergoing a conservative clinical treatment that restricts overexertion.

The majority of clinical studies report trastuzumab-induced cardiotoxicity as a rare event, and, when present, characterized by mild to moderate clinical signs, the ease of reversibility with pharmacological measures and the temporary discontinuation of the medication. Conversely, it is vital for the oncologist/cardiologist to consider the possibility that trastuzumab-induced cardiotoxicity may manifest itself as a severe clinical case, mimicking acute coronary syndrome, justifying careful risk stratification and adequate cardiac monitoring, especially in high-risk patients.
\end{abstract}

Trastuzumab is an important biological agent in the treatment of HER2-positive breast cancer, with effects on response rates, progression-free survival, overall survival and quality of life. The drug was approved by the United States Food and Drug Administration in 1998 for the treatment of metastatic breast cancer, and in 2006, its use was expanded for treatment of adjuvant HER2-positive breast cancer. The 
Herceptin Adjuvant (HERA), National Surgical Adjuvant Breast and Bowel Project B-31 (NSABP B-31), North Central Cancer Treatment Group N9831 (NCCTG N9831), Breast Cancer International Research Group 006 (BCIRG 006) and Finland Herceptin (FinHER) clinical trials showed that adding trastuzumab to standard chemotherapy confers a gain of approximately $50 \%$ in the reduction of recurrence and of $33 \%$ in the reduction of risk of death [1-5].

Although this drug is well tolerated in terms of adverse effects, trastuzumabassociated myocardiotoxicity has been described to have an incidence of $0.6-4.5 \%$, which is reversible with the discontinuation of the medication $[6,7]$. In rare cases, the drug can trigger severe congestive heart failure with progression to death or even mimic acute coronary syndrome with complete left bundle branch blockade [8]. Myocardiotoxicity can be manifested through progressive heart failure (HF) symptoms; the risk of severe HF was found to be $0.6 \%$ in the HERA study and $4.1 \%$ in the NSABP B-31 study $[1,2]$.

Here, we report a case of trastuzumab-associated myocardiotoxicity manifesting as acute coronary syndrome (ACS) in a 69-year-old female. The patient had a history of well-controlled systemic arterial hypertension and diabetes mellitus and a diagnosis of locally advanced invasive ductal carcinoma of the right breast (T4bN3M0), with positive hormone and HER2 receptors. Neoadjuvant chemotherapy was performed with six cycles of docetaxel and epirubicin (accumulated dose of epirubicin of 337 $\mathrm{mg} / \mathrm{m}^{2}$ ), with a partial response according to RECIST. The patient was subjected to a mastectomy with axillary dissection, followed by radiotherapy of the plastron and supraclavicular fossa. Radiotherapy was followed with hormone therapy with letrozole at $2.5 \mathrm{mg} /$ day, combined with trastuzumab at an initial dose of $8 \mathrm{mg} / \mathrm{kg}(634 \mathrm{mg}$ ) and a maintenance dose of $6 \mathrm{mg} / \mathrm{kg}$ ( $468 \mathrm{mg}$ ). Eleven trastuzumab infusions were performed, with the last being conducted 15 days before hospitalization. The patient was admitted with complaints of progressive dyspnea for 1 week, with profound deterioration in the previous hour. At the physical exam, the patient had shortness of breath and labored breathing, with a respiratory rate of 24 , bilateral crackles up to the lung apices, oxygen saturation of $72 \%$ in room air, heart rate of 126 and blood pressure of $170 / 110 \mathrm{~mm} \mathrm{Hg}$. After diagnosing acute pulmonary edema, noninvasive mechanical ventilation and intravenous nitroglycerin were administered. An electrocardiogram (ECG) was performed, showing an advanced left bundle branch block (LBBB) that was nonexistent in a previous examination (fig. 1). Myocardial necrosis markers were high (CK-MB: 52 $\mathrm{U} / \mathrm{l}$; troponin I: $1.66 \mu \mathrm{g} / \mathrm{l})$, as was NT-proBNP $(3,914 \mathrm{pg} / \mathrm{ml})$. Although the patient had a normal examination dated 2 months earlier, her echocardiogram (echo) showed markedly reduced left ventricular systolic performance (LVEF: 15\%) with diffuse hypokinesia and apical thrombus (table 1). Measures for ACS were initiated (aspirin, clopidogrel and enoxaparin), and coronary angiography was performed, which revealed coronary arteries without signs of obstruction. ACS evolved with the disappearance of LBBB on the ECG and the counteraction of clinical symptoms with diuretics, angiotensin-converting enzyme (ACE) inhibitors and beta-blockers.

The clinical and laboratory abnormalities observed were attributed to trastuzumabrelated cardiotoxicity (fig. 2 ). The patient was discharged with medications for $\mathrm{HF}$ in combination with oral anticoagulants. At the 30-day follow-up, the patient underwent a new echo, showing $25 \%$ LVEF associated with significant improvement of dyspnea symptoms (fig. 3 ). Trastuzumab treatment was discontinued and permanently 
contraindicated. The patient is currently undergoing a conservative clinical treatment that restricts overexertion. Trastuzumab is a humanized monoclonal antibody that selectively binds to the extracellular domain of the HER2 receptor, a product of the gene $E R B B 2$. Its mechanism of action is not completely understood; however, it is known that immune responses are involved as well as inhibition of HER2 cleavage, inhibition of PI3K and angiogenesis and possible stabilization of cells in the G1 phase of the cell cycle $[9,10]$. In metastatic disease, trastuzumab provides significant clinical benefit, with an increase of 8.5 months in overall survival when combined with docetaxel chemotherapy compared to chemotherapy treatment alone $[11,10]$. The use of trastuzumab in adjuvant treatment reduces the risk of recurrence in approximately $50 \%$ and the mortality rate by approximately $30 \%$ of patients $[10,12,13]$. In the neoadjuvant setting, the addition of trastuzumab has provided a complete pathological response rate of $66.7 \%$, which is significantly higher than the $25 \%$ found with chemotherapy treatment alone $[10,14]$.

Trastuzumab has been associated with the development of cardiomyopathy, most often manifesting with asymptomatic reduction of LVEF and less frequently with the presence of clinical signs of HF and arrhythmias. This association includes the possibility of cardiac intramural thrombus formation and acute myocardial infarction [15-17].

The pathophysiology of trastuzumab-related myocardial dysfunction has not been well defined, but the following mechanisms have been proposed: drug-drug interaction; immune-mediated destruction of cardiomyocytes; defective HER2 signaling and signaling for the opening of calcium channels, which are important for the maintenance of cardiac contractility; indirect consequences of extracardiac effects of trastuzumab and reduction of HER2-mediated cardiomyocyte survival through the hypoexpression of HER2 [17]. Cardiac dysfunction induced by trastuzumab appears to be primarily due to the inhibition of HER2 signaling in cardiomyocytes, which is associated with changes in the expression of BCL-X proteins, ATP depletion and consequent contractile dysfunction but without causing changes in cellular structure $[10,18]$. This phenomenon is a cardiac dysfunction that has no correlation with lesions in myocardial cells, with only functional and without morphological effects.

The inhibition of HER2-mediated pathways caused by trastuzumab prevents the response of cardiomyocytes to cardiac overload factors, interfering with gp130 cytokine and neuregulin signaling, altering the maintenance mechanisms of myocardial viability and allowing the onset of cardiac dysfunction [10, 19]. HER2 expression is important for embryonic cardiac development and cardioprotection, modulating the cardiac response to stress. Mutation of the HER2 gene in animal models leads to early death by dilated cardiomyopathy and greater susceptibility to cellular death when exposed to anthracyclines. Reduced expression of HER2 may lead to dilated cardiomyopathy similar to dilated cardiomyopathy in humans $[10,20]$. Through the activation of HER2 and ErbB4, neuregulin promotes the hypertrophy of adult cardiomyocytes and the proliferation of embryonic cardiomyocytes, protecting them from apoptosis [16]. In animal models, neuregulin treatment improves cardiac performance and increases survival in animals with cardiomyopathy induced by heart attack, drugs or myocarditis, suggesting the possibility of using this substance as a treatment for dilated cardiomyopathy [10]. 
$\mathrm{HF}$ occurs in $1-4 \%$ of patients treated with trastuzumab. In approximately $10 \%$ of patients, there is a reduction in cardiac function that is reversible in the majority of cases. HF has been described both from the use of trastuzumab alone (4\%) and in combination (27\%) with other antineoplastic agents, such as anthracyclines and cyclophosphamide. The risk of cardiac dysfunction associated with the use of trastuzumab in this circumstance is justified by the $25 \%$ gain in overall survival, but it must be emphasized that combining these therapies increases the risk of cardiac dysfunction in up to $19 \%$ of patients when associated with regimens that include doxorubicin and cyclophosphamide [2, 3]. In 2005, the Cardiac Review and Evaluation Committee (CREC) standardized the criteria for diagnosing cardiac events/dysfunction and categorized the events in accordance with the New York Heart Association standards. The CREC criteria for diagnosing cardiac dysfunction are cardiomyopathy characterized by a decrease in LVEF with global or more severe dysfunction in the septum; severe congestive heart failure; associated signs of HF, including gallop, tachycardia or both; a decrease of at least $5 \%$ and up to 50\% in LVEF, followed by signs and symptoms of CHF or a decrease of at least $10 \%$ and up to $55 \%$ without signs of symptoms [2, 3]. Data from the NSABP B-31 study, reevaluated according to these criteria, showed that among the 31 patients who were given trastuzumab and had developed a major cardiac event, 28 developed this cardiac event during trastuzumab therapy, and only 3 had subsequent cardiac events after completing the 1-year therapy. Of these 31 patients who had a CHF diagnosis, 27 remained under observation for at least 6 months after the diagnosis, and 26 of them remained asymptomatic during this period. Of these 26 patients, 18 (67\%) continued to receive medication for HF. Finally, 24 of the 27 patients who had remained under observation had LVEF measured over the 6-month period following HF diagnosis, and 17 of the 24 patients maintained LVEF below baseline (25 to $69 \%$ ) [2, 3].

Analysis of the cumulative risk of cardiotoxicity in the NSABP B-31 and NCCTG N9831 studies was impaired due to the short follow-up time and cardiac monitoring, in the protocol-specific definition of heart failure, in data collection and in the data review process. After analyzing these studies using the CREC criteria, it was found that cardiac events occurred in $2 \%$ of the patients given trastuzumab and in $0.45 \%$ of patients who only received chemotherapy after a median follow-up of 2 and 1.9 years, respectively. Based on data from the NSABP B-31 study, 1 out of every 30 women treated with trastuzumab will develop a cardiac event, defined as cardiac death or severe HF in 3 years. Furthermore, one in every five women treated with trastuzumab will develop some form of cardiac dysfunction that requires the discontinuation of treatment $[1,3]$.

In the BCIRG 006 study, the LVEF of $17.3 \%$ of the 1,040 patients treated with the adriamycin, cyclophosphamide, taxol, herceptin (ACTH) regimen (table 1) sustained a drop of over $10 \%$ from baseline after treatment with trastuzumab. In addition to HF, cardiac events were also identified in the form of myocardial ischemia and arrhythmias. The overall rate of clinically significant cardiac events (grade $3 / 4$ ) was $0.95 \%$ for ACT and $2.3 \%$ for ACTH ( $\mathrm{p}=0.016)$, showing increased toxicity with the combination that included trastuzumab [4].

The CREC analyzed the risk factors in a univariate analysis for the development of a cardiac event. The analysis included the use of trastuzumab (4.38-fold increased risk), age over 50 years (2.84-fold increased risk) and low LVEF at the start of the paclitaxel regimen and at the end of the doxorubicin regimen $(p=0.0106)$. The risk increased 
from $0.6 \%$ for patients without any risk factors to $5.2 \%$ for the trastuzumab group, whereas for chemotherapy-only treatment, this risk increased from 0.3 to $2.4 \%$ [1, 2].

In 2007, as a result of the update of the NSABP B-31 study results, a predictive model was proposed for the occurrence of cardiac events in patients previously treated with anthracycline and trastuzumab, analyzing criteria such as age, LVEF and the presence of systemic arterial hypertension (SAH) during trastuzumab treatment [21].

$$
\{[7.4+(0.03 \times \text { age })-(0.1 \times \text { LVEF })+0.68 \text { se } \mathrm{SAH}] \times 100\} / 4.82
$$

Previous or concomitant use of anthracycline (cumulative dose of $400 \mathrm{mg} / \mathrm{m}^{2}$ ) or taxanes, chest wall irradiation, antihypertensive therapy and preexisting cardiac dysfunction are additional risk factors for cardiac dysfunction, in addition to obesity and age over 60 years.

Conversely, valvular heart disease and type 2 diabetes mellitus do not significantly increase the risk of cardiotoxicity using multivariate analysis $[3,6,7]$. The patient from our case report had the risk factors of age over 60 years, hypertension, diabetes and grade I obesity, and she had undergone radiotherapy and was not given ACE inhibitors to control SAH. The patient had also received a cumulative dose of epirubicin of 337 $\mathrm{mg} / \mathrm{m}^{2}$. Therefore, she was at high risk for developing cardiotoxicity resulting in cardiomyopathy.

Although patients with SAH and inadequate blood pressure control were excluded from the trastuzumab trial, patients with controlled SAH were given randomized treatment regimens. In NSABP B-31, approximately $20 \%$ of patients randomized for trastuzumab use were given some type of antihypertensive medication concomitantly. It is possible that many of these patients were already using ACE inhibitors and/or beta-blockers, which both favorably alter the natural course of left ventricle dysfunction. In this context, it is possible that the use of these agents favorably influenced the low cumulative incidence of cardiotoxicity observed $[2,22]$.

In the management of cardiotoxicity by trastuzumab, it remains unknown whether there are any benefits to introducing ACE inhibitors, which have been shown to be effective in slowing the progression of left ventricle cardiac dysfunction in several different clinical scenarios such as anthracycline-induced cardiomyopathy. In this sense, it is likely that in high-risk patients, prophylactic strategies based on ACE inhibitors are able to prevent certain cardiotoxicity events related to medications [22].

The factors identified in these analyses can help in the risk stratification of individual patients to develop a cardiac event. Nevertheless, it is still not possible to identify with any certainty the population of patients who will experience a cardiac event related to trastuzumab treatment or to identify an exact value for LVEF that characterizes an increased risk [1,3]. Conversely, there are specific recommendations for monitoring cardiac function during trastuzumab treatment and guidelines on suspension or maintenance of treatment according to changes in the cardiac ejection fraction. Typically, LVEF is the reference standard for cardiac function monitoring, but this technique has less sensitivity in addition to being examiner-dependent. New monitoring methods such as NT-proBNP and troponin I have been used as validated tools and in the early identification of cardiac damage [21-23]. 
Based on the HERA study, which had a 3.6-year follow-up period, assessment of cardiotoxicity was proposed. This assessment included cardiac monitoring, a questionnaire, a physical exam and an ECG during the first consultation in addition to LVEF assessment by echo or scintigraphy of the heart chambers (multigated acquisition heart scan) at 3, 6, 12, 18, 24, 30, 36 and 48 months and annually between 5 and 10 years $[1,23]$.

Currently, real-time 3D transthoracic echo is a viable method to accurately assess the changes in the values of LV volume and LVEF and also to serially monitor patients receiving chemotherapy with anthracyclines or trastuzumab [23].

Among the noninvasive imaging modalities, magnetic resonance imaging is the most accurate and reproducible because it can detect subtle changes in LVEF; however, due to its high cost, low availability and the need for a trained specialist for its interpretation, this technique has not been used to monitor cardiotoxicity in clinical studies [23]. In clinical practice, the choice of imaging method is directly related to the availability of resources of the healthcare center.

The majority of clinical studies report trastuzumab-induced cardiotoxicity as a rare event, and, when present, characterized by mild to moderate clinical signs, the ease of reversibility with pharmacological measures and the temporary discontinuation of the medication. Conversely, it is vital for the oncologist/cardiologist to consider the possibility that trastuzumab-induced cardiotoxicity may manifest itself as a severe clinical case, mimicking ACS, justifying careful risk stratification and adequate cardiac monitoring, especially in high-risk patients.

Table 1. Treatment protocols of clinical studies that used trastuzumab for breast cancer

\begin{tabular}{|c|c|c|c|c|}
\hline NSABP B-31 & NCCTG N9831 & HERA & BCIRG 006 & FinHER \\
\hline $\begin{array}{l}\text { AC every } 21 \text { days for } 4 \\
\text { cycles }+ \text { Pac every } 21 \\
\text { days for } 4 \text { cycles }\end{array}$ & $\begin{array}{l}\text { AC every } 21 \text { days for } 4 \\
\text { cycles + weekly PAC for } 12 \\
\text { cycles }\end{array}$ & $\begin{array}{l}4 \text { or more QT cycles + } \\
\text { observation }\end{array}$ & $\begin{array}{l}\text { AC every } 21 \text { days for } 4 \text { cycles } \\
\text { + Doc every } 21 \text { days for } 4 \\
\text { cycles }\end{array}$ & $\begin{array}{l}\text { Doc every } 21 \text { days for } 3 \\
\text { cycles + weekly TZM for } 9 \\
\text { cycles + FEC for } 3 \text { cycles }\end{array}$ \\
\hline \multirow[t]{3}{*}{$\begin{array}{l}\text { AC every } 21 \text { days for } 4 \\
\text { cycles }+ \text { Pac every } 21 \\
\text { days for } 4 \text { cycles }+ \\
\text { weekly TZM for } 1 \text { year }\end{array}$} & $\begin{array}{l}\text { AC every } 21 \text { days for } 4 \\
\text { cycles }>\text { Pac without for } 12 \\
\text { cycles }>\text { TZM without for } 1 \\
\text { year }\end{array}$ & $\begin{array}{l}4 \text { or more QT cycles + } \\
\text { TZM every } 21 \text { days for } \\
1 \text { year }\end{array}$ & $\begin{array}{l}\text { AC every } 21 \text { days for } 4 \text { cycles } \\
\text { + Doc every } 21 \text { days for } 4 \\
\text { cycles + weekly TZM for } 12 \\
\text { weeks + TZM every } 21 \text { days } \\
\text { for } 40 \text { weeks for } 1 \text { year }\end{array}$ & $\begin{array}{l}\text { Doc every } 21 \text { days for } 3 \\
\text { cycles + FEC for } 3 \text { cycles }\end{array}$ \\
\hline & $\begin{array}{l}\text { AC every } 21 \text { days for } 4 \\
\text { cycles >Pac without for } 12 \\
\text { cycles + TZM without for } 1 \\
\text { year }\end{array}$ & $\begin{array}{l}4 \text { or more QT cycles + } \\
\text { TZM every } 21 \text { days for } \\
2 \text { years }\end{array}$ & $\begin{array}{l}\text { Doc + Cap every } 21 \text { days for } 6 \\
\text { cycles + weekly TZM for } 18 \\
\text { weeks + every } 21 \text { days for } 1 \\
\text { year }\end{array}$ & $\begin{array}{l}\text { Vin every } 21 \text { days for } 3 \\
\text { cycles + weekly TZM for } 9 \\
\text { cycles + FEC for } 3 \text { cycles }\end{array}$ \\
\hline & & & & $\begin{array}{l}\text { Vin every } 21 \text { days for } 3 \\
\text { cycles + FEC for } 3 \text { cycles }\end{array}$ \\
\hline
\end{tabular}

NSABP = National Surgical Adjuvant Breast and Bowel Project; NCCTG $=$ North Central Cancer Treatment Group; HERA = Herceptin Adjuvant trial; $\mathrm{AC}=$ doxorubicin (Adriamycin) and cyclophosphamide; $\mathrm{BCIRG}=$ Breast Cancer International Research Group; FinHER = Finland Herceptin trial; FEC = fluorouracil, epirubicin, cyclophosphamide; Pac = paclitaxel; TZM = trastuzumab; Cap = carboplatin; Doc = docetaxel; Vin = vinorelbine. 

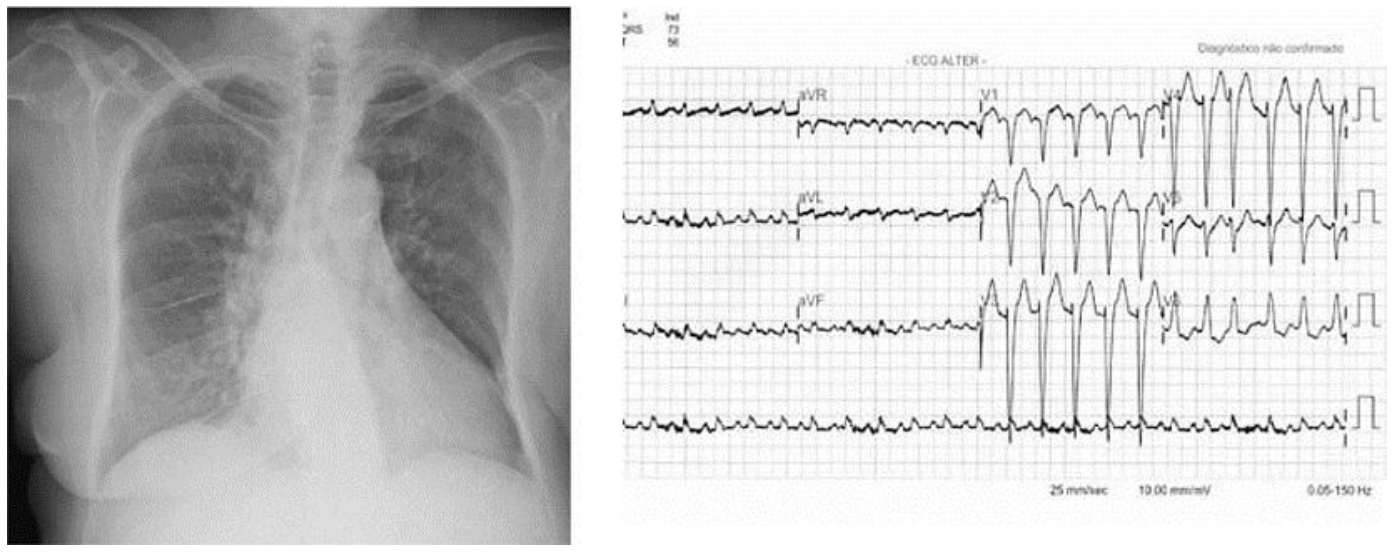

Fig. 1. Chest radiograph with cardiomegaly and pulmonary congestion. ECG with sinus tachycardia, advanced LBBB and ST-segment elevation on the anterior wall of the myocardium (July 13, 2010).
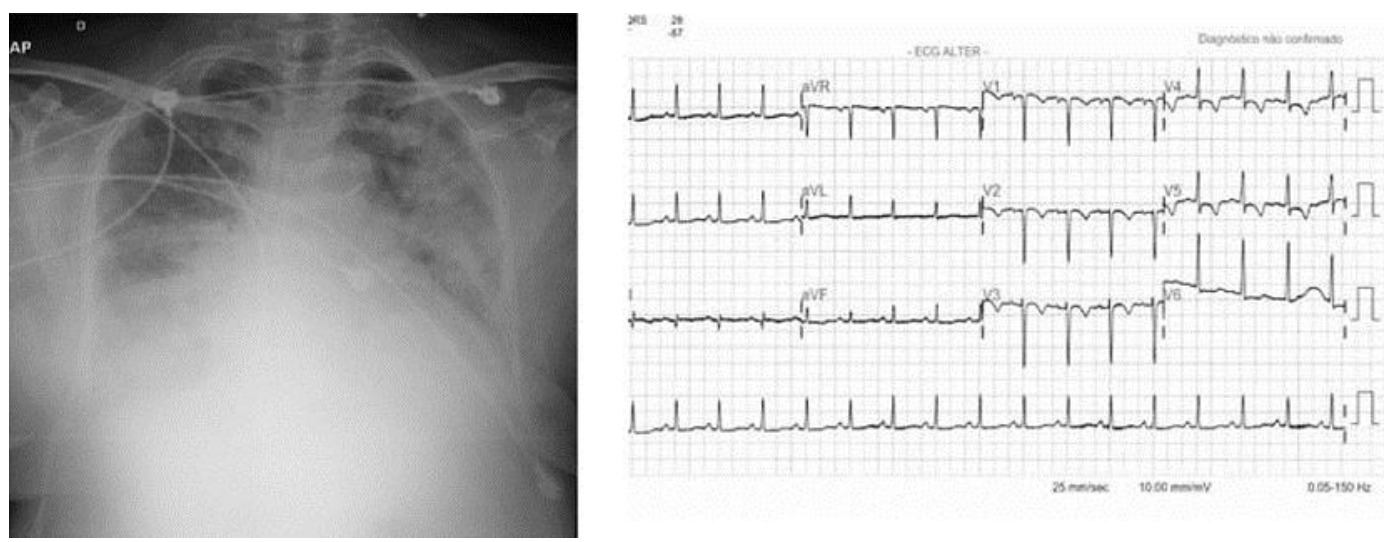

Fig. 2. Chest radiograph showing cardiomegaly without pulmonary congestion. ECG with sinus rhythm, HR of approximately $100 \mathrm{bpm}$ and a lesion on the anterior wall (July 16, 2010).

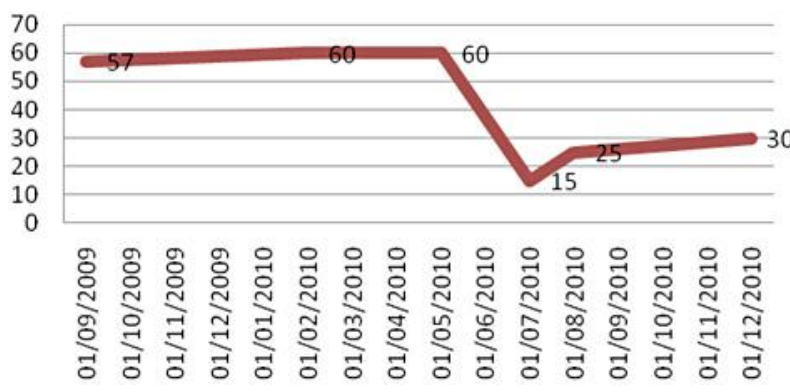

Fig. 3. Temporal correlation of LVEF. 


\section{References}

$\checkmark 1$ Procter M, Suter TM, de Azambuja E, Dafni U, van Dooren V, Muehlbauer S, Climent MA, Rechberger E, Liu WT, Toi M, Coombes RC, Dodwell D, Pagani O, Madrid J, Hall M, Chen SC, Focan C, Muschol M, van Veldhuisen DJ, Piccart-Gebhart MJ: Longer-term assessment of trastuzumab-related cardiac adverse events in the Herceptin Adjuvant (HERA) trial. J Clin Oncol 2010;28:3422-3428.

-2 Telli ML, Hunt SA, Carlson RW, Guardino AE: Trastuzumab-related cardiotoxicity: calling into question the concept of reversibility. J Clin Oncol 2007;25:3525-3533.

-3 Russell SD, Blackwell KL, Lawrence J, Pippen JE Jr, Roe MT, Wood F, Paton V, Holmgren E, Mahaffey KW: Independent adjudication of symptomatic heart failure with the use of doxorubicin and cyclophosphamide followed by trastuzumab adjuvant therapy: a combined review of cardiac data from the National Surgical Adjuvant breast and Bowel Project B-31 and the North Central Cancer Treatment Group N9831 clinical trials. J Clin Oncol 2010;28:3416-3421.

-4 Nabholtz JM, Reese DM, Lindsay MA, Riva A: HER2-positive breast cancer: update on Breast Cancer International Research Group trials. Clin Breast Cancer 2002;3(suppl 2):S75-S79.

5 Costa RB, Kurra G, Greenberg L, Geyer CE: Efficacy and cardiac safety of adjuvant trastuzumab-based chemotherapy regimens for HER2-positive early breast cancer. Ann Oncol 2010;21:2153-2160.

6 Floyd JD, Nguyen DT, Lobins RL, Bashir Q, Doll DC, Perry MC: Cardiotoxicity of cancer therapy. J Clin Oncol 2005;23:7685-7696.

7 Orphanos GS, Ioannidis GN, Ardavanis AG: Cardiotoxicity induced by tyrosine kinase inhibitors. Acta Oncol 2009;48:964-970.

-8 Tu CM, Chu KM, Yang SP, Cheng SM, Wang WB: Trastuzumab (Herceptin)-associated cardiomyopathy presented as new onset of complete left bundle-branch block mimicking acute coronary syndrome: a case report and literature review. Am J Emerg Med 2009;27:903.e1-3.

9 Valabrega G, Montemurro F, Aglietta M: Trastuzumab: mechanism of action, resistance and future perspectives in HER2-overexpressing breast cancer. Ann Oncol 2007;18:977-984.

10 Sanches SM, Silva JM: Interação entre especialidades: miocardiopatia dilatada e neoplasia de mama HER2 positiva. Arq Bras Cardiol 2010;94:e11-e15.

11 Marty M, Cognetti F, Maraninchi D, Snyder R, Mauriac L, Tubíana-Hulin M, et al: Randomized phase Il trial of the efficacy and safety of trastuzumab combined with docetaxel in patients with human epidermal growth factor receptor 2-positive metastatic breast cancer administered as first-line treatment: the M77001 study group. J Clin Oncol 2005;23:4265-4274.

12 Piccart-Gebhart MJ, Procter M, Leyland-Jones D: Trastuzumab after adjuvant chemotherapy in HER2 positive breast cancer. N Engl J Med 2005;353:1661-1672.

$\checkmark 13$ Romond E, Perez E, Bryant J: Trastuzumab plus adjuvant chemotherapy for operable HER2 positive breast cancer. N Engl J Med 2005;353:1673-1684.

14 Buzdar AU, Ibrahim NK, Francis D, Booser DJ, Thomas, ES, Theriault RL, et al: Significantly higher pathologic complete remission rate after neoadjuvant therapy with trastuzumab, paclitaxel, and epirrubicin chemotherapy: results of a randomized trial in human epidermal growth factor receptor 2positive operable breast cancer. J Clin Oncol 2005;23:3676-3685.

15 Solimando DA Jr: Lexi-Comp Drug Information Handbook for Oncology. A Complete Guide to Combination Chemotherapy Regimens, ed 8. Hudson, Lexi-Comp, 2010, pp 1136-1141.

16 FDA. http://www.accessdata.fda.gov/drugsatfda_docs/label/2002/trasgen082802LB.pdf

17 Perez EA, Morgan JP: Cardiotoxicity of trastuzumab. UpToDate Online 2010. Last literature review version 18.2. http://www.uptodate.com/online.

18 Force T, Krause DS, Van Etten RA: Molecular mechanisms of cardiotoxicity of tyrosine kinase inhibition. Nature 2007;7:332-343.

19 Chien KR: Hercetin and the heart - a molecular modifier of cardiac failure. N Engl J Med 2006;354:789790.

20 Crone SA, Zhao YY, Fan L: ErbB2 is essential in the prevention of dilated cardiomyopathy. Nat Med 2002;8:459-465.

21 Fiúza M: Cardiotoxicity associated with trastuzumab treatment of HER2+ breast cancer. Adv Ther 2009;26(suppl 1):S9-S17.

22 Cardinale D, Colombo A, Sandri MT, Lamantia G, Colombo N, Civelli M, Martinelli G, Veglia F, Fiorentini C, Cipolla CM: Prevention of high-dose chemotherapy-induced cardiotoxicity in high-risk patients by angiotensin-converting enzyme inhibition. Circulation 2006;114:2474-2481. 
-23 Walker J, Bhullar N, Fallah-Rad N, Lytwyn M, Golian M, Fang T, Summers AR, Singal PK, Barac I, Kirkpatrick ID, Jassal DS: Role of three-dimensional echocardiography in breast cancer: comparison with two-dimensional echocardiography, multiple-gated acquisition scans, and cardiac magnetic resonance imaging. J Clin Oncol 2010;28:3429-3436. 